\title{
Analyse Par Approche Hydrogeochimique De La Distribution Spatiale De La Salinite Des Eaux De La Nappe Et Des Sables Quaternaires Du Secteur Des Niayes Du Gandiolais (Littoral Nord Senegalais)
}

\author{
Souleymane Niang, \\ Laboratoire LEIDI, Université Gaston Berger de Saint-Louis \\ Amadou Abou Sy, \\ Université Cheikh Anta DIOP de Dakar, département de géographie \\ Cheikh Ahmed Tidiane Faye, \\ Seydou Alassane Sow, \\ Boubou Aldiouma Sy, \\ Laboratoire LEIDI, Université Gaston Berger de Saint-Louis
}

Doi:10.19044/esj.2020.v16n24p104 URL:http://dx.doi.org/10.19044/esj.2020.v16n24p104

\section{Résumé}

Dans le Gandiolais, la nappe des sables quaternaires, fortement salée sur la majeure partie de la zone, est utilisée pour irriguer les cultures et pose de véritables difficultés pour la durabilité du système maraicher. La salinisation secondaire du sol irrigué et la réaction du complexe absorbant dépendent principalement des paramètres intrinsèques, particulièrement la texture du sol.

L’objet de cet article est d'analyser la distribution spatiale de la salinité en tenant en compte les caractéristiques granulométriques du complexe absorbant. L'approche hydrogéochimique basée sur les relations de concentration permet d'apprécier les modalités de conservation in situ entre les eaux d'irrigation et le sol exploité. Les résultats obtenus ont confirmé que la texture grossière de type sables moyens à fins permet un lessivage rapide des sels solubles sur tout le profil. Les sols restent globalement calcimagnésiens même si les eaux d'irrigation sont globalement chlorurées sodiques et potassiques ou sulfatées sodiques. Cependant, cette forte perméabilité du complexe constitue un risque de surconcentration ionique dans la nappe. Les retours d'irrigation associés aux engrais accentuent la minéralisation de la nappe des sables quaternaires.

Mots-Clés : Hydrogéochimie, Irrigation, Nappe, Salinisation, Gandiolais, Sénégal. 


\title{
Analysis By Hydrogeochemical Approach Of The Spatial Distribution Of The Salinity Of Groundwater And Quaternary Sands Of The Gandiolais Sector (Northern Senegalese Coast)
}

\author{
Souleymane Niang, \\ Laboratoire LEIDI, Université Gaston Berger de Saint-Louis \\ Amadou Abou Sy, \\ Université Cheikh Anta DIOP de Dakar, département de géographie \\ Cheikh Ahmed Tidiane Faye, \\ Seydou Alassane Sow, \\ Boubou Aldiouma Sy, \\ Laboratoire LEIDI, Université Gaston Berger de Saint-Louis
}

\begin{abstract}
In the Gandiolais, the groundwater of the quaternary sands, heavily salted over most of the area, is used to irrigate crops and poses real difficulties for the sustainability of the market gardening system. Secondary salinization of the irrigated soil and the reaction of the absorbing complex depend mainly on the intrinsic parameters, particularly the texture of the soil.

The purpose of this article is to analyze the spatial distribution of salinity taking into account the particle size characteristics of the absorbent complex.The hydrogeochemical approach based on the relations of concentration makes it possible to appreciate the process of conservation in situ between the irrigation water and the exploited soil. The results obtained confirmed that the coarse texture of medium-to-fine sand type allows rapid leaching of soluble salts over the entire profile. Soils remain globally calcimagnesian although the irrigation water is globally sodium chloride and potassium or sodium sulphated. However, this high permeability of the complex constitutes a risk of ionic overconcentration in the aquifer. Irrigation returns associated with fertilizers accentuate the mineralization of the groundwater of the quaternary sands.
\end{abstract}

Kewords : Hydrogeochemistry, Irrigation, Groundwater, Salinization, Gandiolais, Senegal.

\section{I - Introduction}

Le développement agricole en milieu littoral implique, naturellement, 
une vulnérabilité des ressources liées à la physionomie de ces espaces et à des processus inhérents au fonctionnement des littoraux. Sur des espaces côtiers où des activités agricoles sont pratiquées, l'exploitation des ressources souterraines est souvent confrontée à la diffusion du biseau salé soit de manière naturelle (variations des marées ou du marnage, le changement climatique, estuaire inversé), soit elle s'explique par la forte exploitation des ressources souterraines. La salinisation découle des impacts des aménagements dans la modification de la molalité des eaux. L'insuffisance des ressources en eaux de surface et leur irrégularité poussent les populations locales à exploiter souvent les ressources souterraines de qualité médiocre sans réelle conscience de leurs impacts sur le complexe argilo-humique (N. CHKIR, 2008). Or, les échanges entre les eaux salées en apport d'irrigation et le complexe pédologique modifient la qualité agronomique des sols soit en agissant négativement sur la structure, soit en déséquilibrant les échanges cationiques en créant une ambiance ionique asphyxiante (J.Y. LOYER, 1989 ; M. HACHICHA Et Al., 1997 ; S. MARLET Et J.O. JOB, 2006 ; L. KOUZANA，2007 ; K.K. RASSOU, 2009; N. MDIKER, 2009; I. HAMMIA, 2012 ; L. GOUAIDIA Et Al., 2012).

La vulnérabilité aux transferts est à rechercher en premier lieu dans la physionomie du substrat. Les échanges entre le sol en place et les apports d'irrigation sont déterminés par la concentration ionique des eaux mais également par la nature du substrat dans ses phases de lessivage et de capillarité. W. LTIFI (2009) a montré que la texture et la structure du sol agissent bien évidemment sur le processus de la salinisation selon son pouvoir drainant. La chimie des eaux souterraines dépend de la lithologie des terrains traversés et du temps de séjour des eaux (L GOUAIDIA, 2008). La migration des sels est dynamique dans les deux sens : migration en surface par thermocapillarité et migration par lessivage de surface et ou de subsurface.

La figure 1 met en évidence la zone du Gandiolais 




Figure 1 : Localisation du milieu d'étude et des points de mesure de la salinité.

Le Gandiolais appartient à la zone des Niayes longeant tout le littoral Nord du Sénégal. Ces dernières sont des dépressions inter-dunaires (dunes rouges ogoliennes et dunes jaunes semi-fixées) propices aux cultures maraîchères, des espaces de fortes productions horticoles sur la Grande Côte du Sénégal. Il constitue un milieu complexe dans l'estuaire du fleuve Sénégal où les récentes modifications générées par la mise en eau du barrage anti-sel de Diama (1986) et l'ouverture d'une brèche sur la Langue de Barbarie (2003) pour sauver la ville de Saint-Louis des inondations ont sensiblement dégradé les ressources de base notamment la réserve souterraine (I. DIATTA, 2004 ; M.D. DIALLO, 2006 ; A. JACOUTOT, 2006 ; C. KANE, 2010 ; A.A SY, 2013 ; S. NIANG, 2016).

En conséquence, la salinisation actuelle est particulièrement liée au changement du régime salin dans l'estuaire du Sénégal depuis 1986. Si avant le barrage de Diama, les échanges naturels permettaient d'obtenir un régime salin équilibré et répondant aux lois saisonnières de dilution, les récentes modifications (brèche de 2003) ont perturbé cette dynamique de mélange entre les masses d'eau avec une concentration saline importante en aval du barrage de Diama. Ce dernier a réduit l'aire d'extension de la remontée saline. Les eaux marines sont confinées sur la partie aval correspondant, de fait, à l'actuelle zone estuarienne qui semble être délimitée par le barrage en amont. Cependant avec la brèche, les eaux de crue, qui parcouraient le bras du fleuve Sénégal et 
dessalaient l'actuelle lagune de Mboumbaye en l'absence de la rupture, rejoignent directement la mer par bifurcation sur l'extrémité de la rive Nord. Ce qui explique que la partie en aval de la brèche, correspondant à la zone de Gandiol, est constamment salée pendant toute l'année, justifiant la conductivité électrique importante autour de la brèche. Cette dégradation de la qualité de l'eau d'irrigation des cuvettes maraichères du Gandiolais a ainsi entrainé la perte de nombreuses parcelles maraîchères consécutives à des plages de conductivité extrêmement élevées de l'ordre de 10 à 15 ms/cm dans les villages qui sont proche de la lagune de Mboumbaye. Les faciès chimiques identifiés dénotent une origine essentiellement marine. Mais l'irrigation avec ces eaux salées repose essentiellement sur un contenant physique meuble, ce qui modifie les caractéristiques de conservation des ions dans le complexe absorbant.

L'objectif de cette contribution est de montrer, à partir de la caractérisation hydrogéochimique des sols, le rôle des caractéristiques sédimentologiques des cuvettes agricoles dans la dynamique de lessivage et du processus de conservation des sels solubles dans le complexe absorbant sur un horizon de 0 à $30 \mathrm{~cm}$ de profondeur. D'où une méthodologie qui prend en compte à la fois la qualité de l'eau d'irrigation et celle du sol irrigué afin de déterminer la relation de conservation dans la solution.

\section{II - Méthodologie}

Elle repose sur une approche hydrogéochimique basée sur la connaissance du rapport de concentration en ions conservatifs entre l'eau d'irrigation prélevée dans les puits utilisés pour l'irrigation des cuvettes agricoles et les échantillons de sol. La méthode requiert ainsi une estimation du niveau de concentration ionique de l'eau d'irrigation et du sol irrigué. Elle est basée sur la mesure in situ de la conductivité électrique (en mai et octobre 2016) dans les puits d'irrigation et sur des prélèvements d'échantillons d'eaux et de sol. Pour la caractérisation hydrogéochimique, 10 villages de la même toposéquence ont été ciblés pour l'échantillonnage des sols et des eaux d'irrigation afin d'établir le bilan ionique : Bountou Ndour, Ndiébène Gandiol, Gadga Lahrar, Rimbakh Gandiol, Gouye Reine, Gantour, Ndoye Diagne, Pelour 1, Ndeuguette et Ricotte (Fig. 1). Le contraste saisonnier a été privilégié dans le processus d'échantillonnage, le suivi de la salinité et l'analyse des données pour déterminer les variations saisonnières des matières dissoutes dans le sol a été également faite. La conductivité électrique de l'eau d’irrigation à partir des puits utilisés par les maraichers a été mesurée dans 18 villages suivant la toposéquence générale de la côte vers l’intérieur des terres.

En outre, l'étude de la relation entre la salinité et la conservation des sels par l'entrée des caractéristiques intrinsèques du sol repose sur la connaissance des fractions granulométriques. Elle a d'abord consisté à 
échantillonner dans les cuvettes agricoles des villages ciblés afin de déterminer les paramètres lithologiques, la texture du sol notamment (Fig. 1).

Les échantillons d'eau et de sols ont permis de déterminer les paramètres dont le $\mathrm{pH}$ de l'eau, $\mathrm{pH}$ du sol, $\mathrm{Na}^{+}, \mathrm{K}^{+}, \mathrm{Ca}^{+}, \mathrm{Mg}^{+}, \mathrm{Cl}^{-}, \mathrm{SO}_{4}{ }^{2-}$, $\mathrm{HCO}_{3}{ }^{-}, \mathrm{NO}_{3}{ }^{-}$, qui représentent les faciès chimiques : les anions et les cations dans l'eau d'irrigation et la solution du sol. Le calcium $\left(\mathrm{Ca}^{+}\right)$et le magnésium $\left(\mathrm{Mg}^{+}\right)$ont été dosés par volumétrie avec un chélatant ${ }^{1}$; le sodium $\left(\mathrm{Na}^{+}\right)$et le potassium $\left(\mathrm{K}^{+}\right)$sont déterminés par la méthode de spectrophotométrie de flamme ; le dosage des chlorures a été effectué par titrimétrie (méthode de MOHR). L'alcalinité a été déterminée par la méthode volumétrique. Les carbonates et les bicarbonates sont dosés à l'acide sulfurique non concentré $(0$, $02 \mathrm{~N}$ ), à une solution de phénophtaléine dans l'alcool à 0,5\% et d'une solution de méthylorange. Les sulfates ont subi un dosage à la solution de $\mathrm{NaCl}-\mathrm{HCl}$ (10 ml), de chlorure de barium (1 gramme), de gomme d'acacia (2 ml). Les faciès chimiques ont été déterminés par l'approche hydrogéochimique à partir des diagrammes de PIPER et de STABLER. Ce dernier a permis de déterminer, sur un même plan, la prédominance des éléments dissous et d'observer l'effet de conservation dans la relation eau-sol irrigué. La formule caractéristique de STABLER consiste à calculer le pourcentage de chaque ion par rapport à la concentration totale.

La granulométrie a été effectuée selon le système français de normalisation granulométrique (AFNOR) sur des tamis correspondant à une progression géométrique égale à $\sqrt{ } 10 / 10$ avec transformation logarithmique grâce à une substitution par l'échelle arithmétique des $\mathrm{D}\left(\mathrm{D}_{0}=1 \mathrm{~mm}\right)$. Les sédiments ont été tamisés à $2 \mathrm{~mm}$. Les caractéristiques sédimentologiques des sables ont été appréciées par l'analyse et l'interprétation des indices granulométriques et des courbes de distribution. L'échelle des grains est basée sur les études de G.M. FRIEDMAN et F.E. SANDERS (1978) Les résultats granulométriques ont été regroupés en quatre classes : les sables grossiers ( $>$ $2,00 \mathrm{~mm}$ à $<4,75 \mathrm{~mm}$ ), les sables moyens ( $>0,465 \mathrm{~mm}$ à $<2,00 \mathrm{~mm}$ ), les sables fins ( $>0,0625 \mathrm{~mm}$ à $<0,465 \mathrm{~mm})$, les argiles et les limons.

Le couplage de ces données granulométriques associées aux résultats des traitements chimiques ont permis d'aboutir à des conclusions caractéristiques de l'évolution du complexe pédologique irrigué de l'espace agricole du Gandiol.

La cartographie des teneurs a été effectuée sous Arcgis par la méthode d'interpolation spatiale, krigeage.

${ }^{1}$ La chélation est un processus au cours duquel est formé un complexe entre un ligand et un cation métallique alors complexé. 


\section{III - Résultats}

\section{1) Une granulométrie à tendance grossière : facteur de non-conservation des sels dans le complexe absorbant}

Les sols des bas-fonds mis en valeur entrent dans la catégorie des sols hydromorphes de texture essentiellement sableuse. Les sols ne sont pas profonds contrairement à la partie méridionale et centrale du secteur des Niayes. Les sables moyens et fins représentent, en moyenne, 76 à $96 \%$ des échantillons traités avec une dominance de la fraction moyenne, soit 60 à $70 \%$. Les tests sédimentologiques indiquent une nette prédominance des grains moyens et fins avec un renversement du régime sédimentaire dans le secteur continental correspondant aux dunes rouges. Ceci est essentiellement dû à une plus grande importance des particules fines (SF) et très fines (STF) caractéristiques d'un vannage sélectif des sédiments d'où le caractère sigmoïde des courbes de distribution. Les particules sont déplacées à $59 \%$ dans le diamètre $1,5 \mathrm{~mm}$ et $68 \%$ des cas dans le diamètre 1,5 et $2 \%$ dans le diamètre de $2 \mathrm{~mm}$.

Dans les cuvettes littorales (blanches et jaunes semi-fixées), le premier mode est situé à 302,5 $\mu \mathrm{m}(\mathrm{SM})$ et le second mode à 152,5 $\mu \mathrm{m}(\mathrm{SF})$. Ce sont principalement les médianes qui changent légèrement entre les horizons 0-15 et 15-30 mais également entre sites étudiés. Considérant l'horizon 0-15 cm, la médiane se situe respectivement à $284 \mu \mathrm{m}$ (Gadga Lahrar), $275 \mu \mathrm{m}$ (Bountou

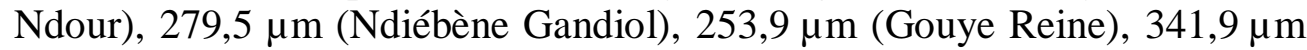
(Rimbakh Gandiol). Pour l'horizon 15-30 cm, les valeurs de la médiane varient sensiblement avec respectivement 285,3 $\mu \mathrm{m}$ (Gadga Lahrar), $272 \mu \mathrm{m}$

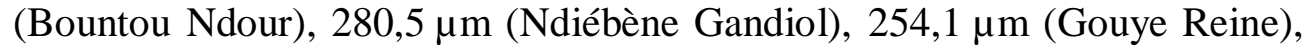
280,5 $\mu \mathrm{m}$ (Rimbakh Gandiol). L'analyse met en évidence deux constats.

Le premier constat montre que la faible différence entre les horizons s'explique par l'homogénéité de la trame texturale et les mêmes conditions de dépôt entre secteur considéré. Le deuxième constat permet d'estimer que le renversement des modes est lié à l'importance des éléments fins dans la composition granulométrique des sables dunaires continentaux.

Également, le profil des cuvettes est toujours sigmoïde et traduit les conséquences du triage sélectif en haut de dunes. Il résulte d'une sélection limitée à une seule tranche de matériel. Les deux extrémités montrent une rapide décroissance traduisant un appauvrissement des particules des tailles correspondantes. Les particules sont déplacées à $59 \%$ dans le diamètre $1,5 \mathrm{~mm}$ et $68 \%$ des cas dans le diamètre 1,5 et $2 \%$ dans le diamètre de $2 \mathrm{~mm}$ (Fig. 2). 


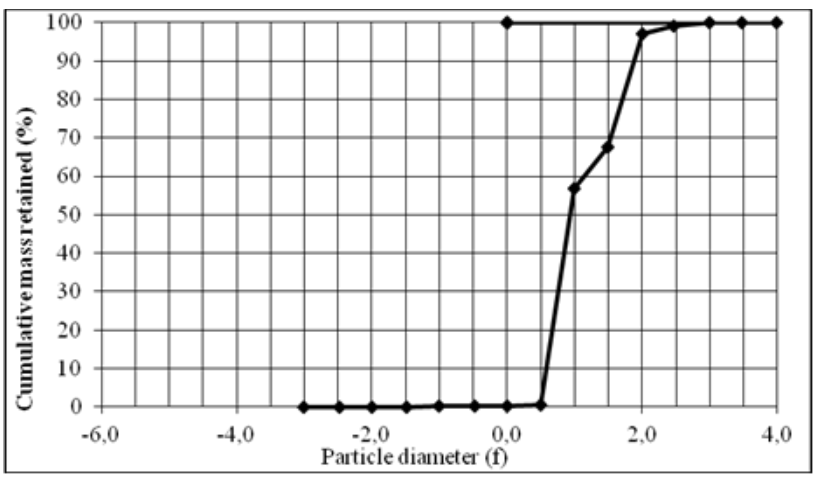

Figure 2 - Courbe cumulée dans les cuvettes

En outre, les horizons présentent des distributions granulométriques similaires. Toutefois, des différences de l'ordre de 1 à $11 \%$ sont observées dans les bas-fonds intérieurs où les horizons $0-15 \mathrm{~cm}$ atteignent 20 à $24 \%$ d'argiles et de limons. Ce qui suppose un pouvoir conservateur des sels plus important dans les niayes typiques que les ndioukis à cause notamment de la présence dans les niayes typiques, des argiles et des limons. Les illustrations (Fig. 3 et 4) mettent en évidence la répartition granulométrique dans les horizons $0-15 \mathrm{~cm}$ et $15-30 \mathrm{~cm}$.

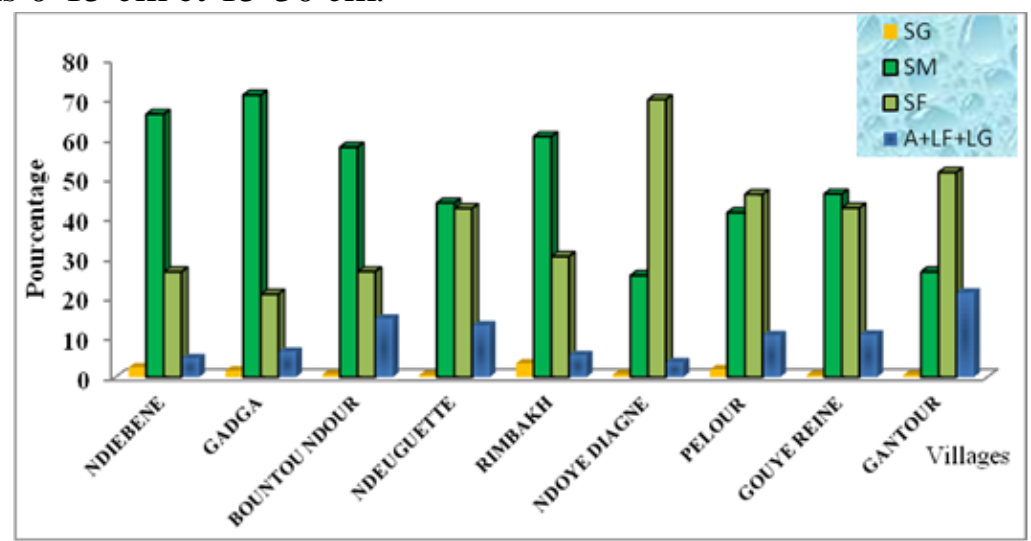

Figure 3 - Analyses granulométriques de l'horizon 0-15 cm dans les niayes du Gandiolais (horizon 0-15 cm)

Les dépressions interdunaires imbriquées dans les dunes littorales sont caractérisées par une prépondérance des sables moyens jusqu'à plus de $70 \%$ (Ndiébène, Bountou Ndour, Gadga Lahrar, Rimbakh, Ricotte, Gouye Reine, Gniling Mbao (figure 3). 




Figure 4 - Analyses granulométriques de l'horizon 15-30 cm dans les niayes du Gandiolais (horizon 15-30 cm)

Globalement, il y a une très faible présence des colloïdes, ce qui a pour effet de favoriser un lessivage important dans les horizons superficiels et en profondeur dès lors que les profils sont homogènes au-delà de $30 \mathrm{~cm}$. Cette tendance grossière des sédiments observée dans les sédiments des niayes, à potentiel gravitaire ${ }^{2}$ très fort et un pouvoir matriciel ${ }^{3}$ très faible, constitue un facteur important de la facilité du lessivage des sels en contact avec la source saline. Les porosités sont comprises entre 34 et $46 \%$ pour une densité comprise entre 1,43 à 1,75. Ces valeurs expliquent la facilité avec laquelle les sels peuvent être lessivés pendant la saison des pluies et dissous dans la nappe des sables quaternaires.

\section{2) Une salinité excessive de la nappe des sables quaternaires}

Les eaux d'irrigation sont caractérisées par de fortes teneurs en sel dues au dynamisme de l'intrusion marine. Ces dernières contiennent entre 4,35 à 15,39 g/l de sel. Les salinités extrêmes correspondent à Ndiébène Gandiol, Mboumbaye, Mouit et Dégou Niaye (situés à moins de $500 \mathrm{~m}$ de la mer). ${ }^{4}$ En saison sèche (octobre à juin), les villages du secteur continental (situés à $9 \mathrm{~km}$ de la mer) enregistrent des CE de l'ordre de $1000 \mu \mathrm{S} / \mathrm{cm}$ à $2000 \mu \mathrm{S} / \mathrm{cm}$, soit une minéralisation globale de 0,83 à 2,07 $\mathrm{g} / \mathrm{l}$ mais qui peuvent se diluer jusqu'à $80 \%$ en fin de saison pluvieuse (septembre).

La salinisation touche pratiquement tout le Gandiolais, mais différemment suivant la distance séparant les localités du biseau salé. Les conductivités de l'eau d'irrigation restent très élevées partout dans les parcelles

\footnotetext{
${ }^{2}$ Exprime l'adaptabilité des sables à l'infiltration des eaux de pluies par gravité.

${ }^{3}$ Exprime la force des sables à retenir l'eau.

${ }^{4}$ Une eau de bonne qualité, destinée au maraîchage, doit avoir une concentration en chlorures évaluée à moins de $600 \mathrm{mg} / \mathrm{l}$. L'eau d'irrigation de bonne qualité contient en principe 200 à $500 \mathrm{mg}$ kg-1 de sel soluble ce qui rejoint globalement les dispositions minéralogiques proposées par la FAO.
} 
maraichères. Et à partir du moment où les valeurs de salinité atteignent $2 \mathrm{~g} / \mathrm{l}$ (Fig. 5), leur utilisation pour l'irrigation devient problématique et accroit l'effet osmose.

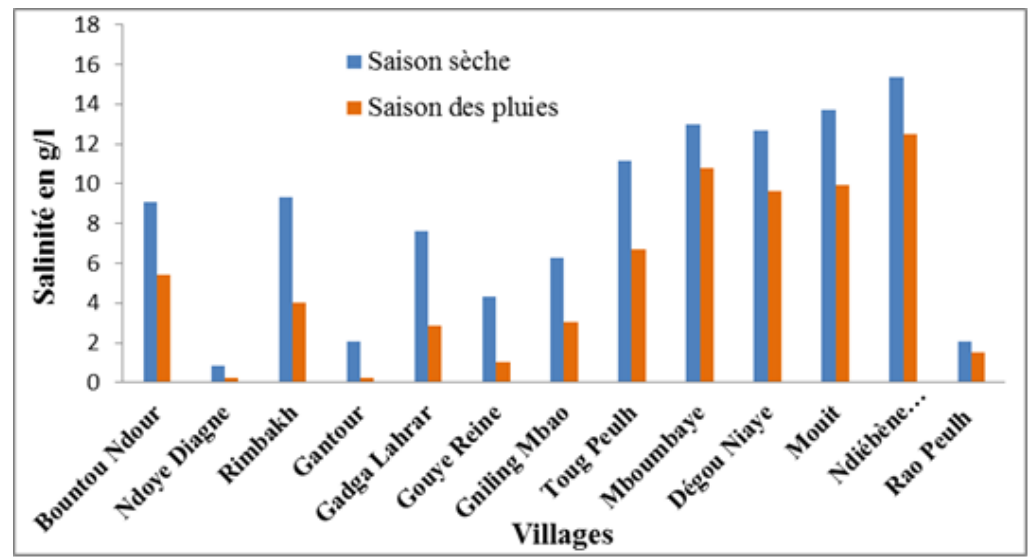

Figure 5 - Variation de la salinité de l'eau en fonction des saisons

Les salinités mesurées à plus de $4 \mathrm{~g} / \mathrm{l}$ correspondent aux sites localisés dans la zone littorale. Les taux de dilution sont de l'ordre de 23,50(Mouit) à $58 \%$ dans le secteur littoral. La dilution dans le secteur central est moyenne à tendance forte 73,93 \% à Gouye Reine et 52,00 \% à Rimbakh Gandiol. Par conséquent, les villages côtiers sont caractérisés par une dilution faible de la nappe avec un pourcentage maximal de 58,03 \% à Gadga Lahrar (Gniling Mbao 45,53 \%, Bountou Ndour 32,80 \%, Mboumbaye 46,43 \% et Mouit 23,50 \%).

Les estimations fournies permettent de dire que les taux de dilution sont plus importants dans le secteur continental et varient entre 85,41 \% à Gantour et $70,28 \%$ à Ndoye Diagne.

Malgré la diminution de la concentration saline des eaux dans le secteur central et au niveau des villages côtiers, les valeurs restent toujours élevées en fin de saison des pluies dans la partie littorale pour en permettre l'irrigation correcte des parcelles maraichères.

Globalement la salinité décroit progressivement vers le continent (Fig. 6). Les conductivités électriques mesurées in situ par un conductimètre au cours des deux saisons restent faibles et décroissent de la lagune de Mboumbaye vers le secteur continental. Les plages de conductivité observées décrivent le sens de migration du biseau salé vers le secteur Est, lequel marque ces influences jusqu'à Toug Wolof situé à 5 km de la mer (Fig. 2). Si à l'heure actuelle, les diverses mesures ont montré que le secteur continental est épargné par la minéralisation progressive de la nappe, il faut aussi souligner que les mêmes mesures permettent de confirmer une tendance au rehaussement des taux de conductivité électrique dans les puits d'irrigation en saison sèche dans la partie continentale à Gantour $(2,07 \mathrm{~g} / \mathrm{l})$, Rao Peul $(1,53 \mathrm{~g} / \mathrm{l})$ et dans les dépressions de Ndeuguette. 


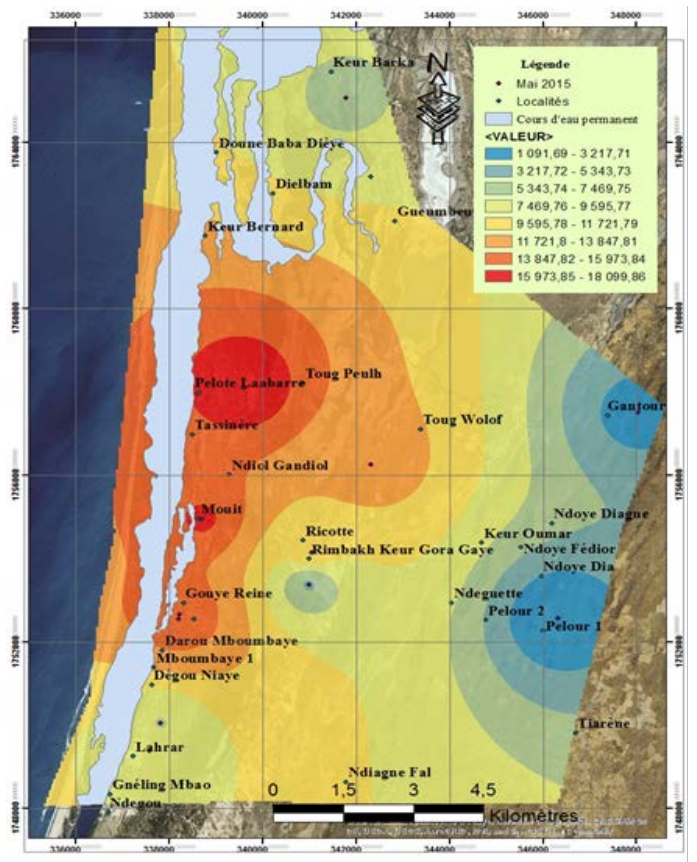

Figure 6: Variation de la salinité de l'eau de la nappe.

Du point de vue hydrochimique, les faciès des eaux d'irrigation (Fig. 7) sont marqués par la présence d'éléments chimiques induisant une intrusion marine sur le continent. Les villages de Rimbakh, de Ricotte, de Toug Peul (zone de transition), de Rao, de Gantour, de Ndoye Dia, de Ndoye Diagne (secteur continental) sont déterminés à $70 \%$ par un faciès chimique chloruré à sulfaté calcique et magnésien. Tandis que Gouye Reine (zone de transition), Mouit, Mboumbaye (secteur plus proche de l'océan) et Gandon indiquent un faciès chimique à $40 \%$ chloruré sodique et potassique ou sulfaté sodique dans les secteurs de fortes concentrations caractérisant des espaces dépressionnaires. Cependant, Mboumbaye tend, tout de même, vers un faciès hyperchloruré sodique au même titre que Mouit et Dégou Niaye.

L'analyse chimique permet de montrer que les cations sont dominés par le sodium alors que dans les anions les chlorures sont partout dominants sauf à Mboumbaye où les sulfates s'associent avec $\mathrm{Na}^{+}$pour former un faciès sulfaté calcique et magnésien. Dans un tel contexte d'extension du biseau salé vers l'intérieur des terres, les faciès passent du type chloruré et sulfaté calcique et magnésien pour devenir des faciès chlorurés sodiques et potassiques ou sulfatés (Fig. 7). 


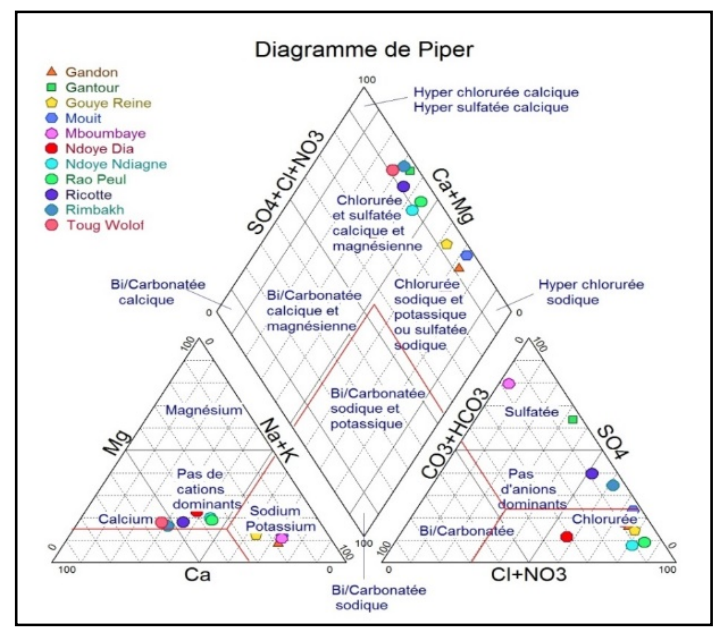

Figure 7 - Faciès chimiques des eaux d'irrigation.

Les faciès chimiques des eaux tendent donc à évoluer vers un enrichissement en sodium et en chlorure. Le développement de ce type de salinité est un indice crédible d'une intrusion saline. Contrairement aux eaux d'irrigation, qui présentent une salinité sévère, les sols des cuvettes irriguées présentent des faciès très différents et moins affectés par la minéralisation.

\section{3) Une faible salinité dans le profil des sols irrigués}

L'analyse de la conductivité permet d'observer une salinité faible dans le profil du sol (Fig. 8) Les valeurs varient respectivement de l'horizon 0-15 cm à 15$30 \mathrm{~cm}$ entre de $700 \mu \mathrm{S} / \mathrm{cm}$ à $205 \mu \mathrm{S} / \mathrm{cm}$ à Gadga Lahrar, de $277 \mu \mathrm{S} / \mathrm{cm}$ à 220 $\mu \mathrm{S} / \mathrm{cm}$ à Bountou Ndour, de 99,4 $\mu \mathrm{S} / \mathrm{cm}$ à 62 us/cm à Keur Dièye, de 664 $\mu \mathrm{S} / \mathrm{cm}$ à 99,5 us/cm à Ndiébène Gandiol, de $73,3 \mu \mathrm{S} / \mathrm{cm}$ à $42,9 \mu \mathrm{S} / \mathrm{cm}$ à Gantour, de 152,7 $\mu \mathrm{S} / \mathrm{cm}$ à $948 \mu \mathrm{S} / \mathrm{cm}$ à Ngaïna, de 27,6 $\mu \mathrm{S} / \mathrm{cm}$ à 22,8 $\mu \mathrm{S} / \mathrm{cm}$, de $53,5 \mu \mathrm{S} / \mathrm{cm}$ à $66,7 \mu \mathrm{S} / \mathrm{cm}$ à Ricotte et de $57,4 \mu \mathrm{S} / \mathrm{cm}$ à $52,4 \mu \mathrm{S} / \mathrm{cm}$ à Rimbakh Gandiol.

Les horizons superficiels $(0-15 \mathrm{~cm})$ sont plus concentrés que les horizons inférieurs $(15-30 \mathrm{~cm})$ mais globalement aucune valeur n'atteint $1 \mathrm{~m} / \mathrm{s}$ exprimant, de fait, une salinité faible dans le sol en saison sèche.

En saison des pluies, la salinité est estimée entre 70 à $118 \mu \mathrm{s} / \mathrm{cm}$ (Gadga Lahrar), 480 à $757 \mu \mathrm{S} / \mathrm{cm}$ (Ndiébène Gandiol), 48 à $405 \mu \mathrm{S} / \mathrm{cm}$ (Pelour), 93 et $209 \mu \mathrm{s} / \mathrm{cm}$ (Rimbakh Gandiol), 19 à $60 \mu \mathrm{S} / \mathrm{cm}$ (Ndoye Diagne), 29 et 113 $\mu \mathrm{s} / \mathrm{cm}$ (Gouye Reine), 15 et $52 \mu \mathrm{S} / \mathrm{cm}$ (Gantour).

Au regard de ces résultats de suivi, les valeurs de salinité du complexe pédologique sont faibles dans les niayes pour en définir un sol salé excepté quelques cuvettes de Ndiébène Gandiol situées autour de sebkhas littorales. La configuration spatiale de la salinité correspond à celle de l'eau de la nappe. La configuration spatiale de la salinité correspond à celle de l'eau de la nappe. 
Considérant la nappe et le sol, la salinité décroit du littoral vers les dunes intérieures (Fig. 8).

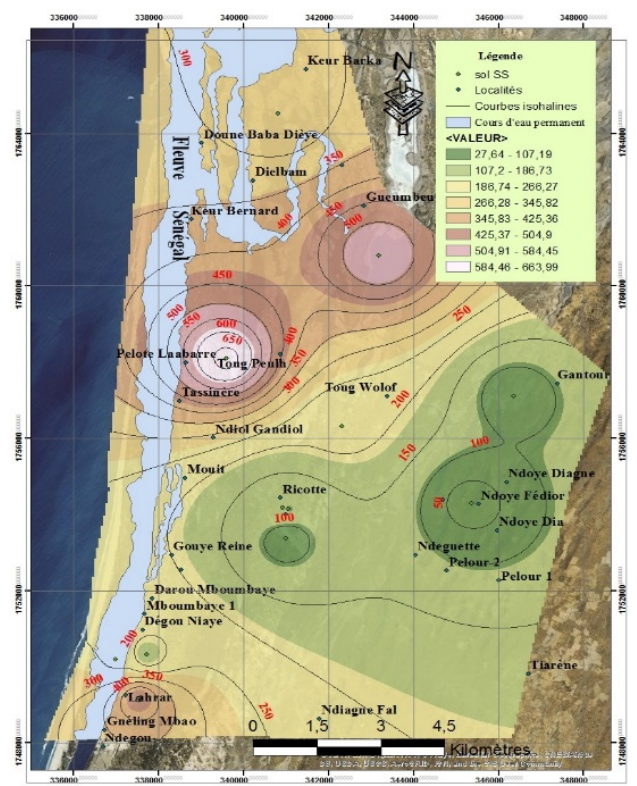

Figure 8 :Variation de la salinité des sols irrigués en saison sèche (SS)

Du point de vue géochimique, la morphologie et les propriétés dynamiques sont marquées par l'abondance d'ions bivalents $\left(\mathrm{Ca}^{+}\right.$et $\left.\mathrm{Mg}^{+}\right)$sous forme de carbonates. Le calcium est estimé entre 2,89 méq/100 g et 1,2 méq/100 dans les dunes littorales et entre 1,50 méq/100 g et 1,37 méq/100 g dans le secteur des dunes rouges moins carbonaté.

En revanche, les teneurs en magnésium sont respectivement de l'ordre de 1,93 méq/100 g à Gadga Lahrar contre 1,52 méq/100 g à Bountou Ndour, 1,31 méq/100 g à Ndiébène Gandiol, 0,63 méq/100 g Gantour, 0,67 méq/100 g à Ndoye Diagne, 1,11 méq/100 g à Rimbakh Gandiol, 1,45 méq/100 g à Gouye Reine, 0,78 méq/100 g à Ndeuguette, 1,36 méq/100 g à Pelour, 0,50 méq/100 $\mathrm{g}$ à Keur Dièye et 0,36 méq/100 g à Ricotte.

Les valeurs de $\mathrm{Na}^{+}$restent comprises entre 0,116 et 0,676 méq/100 g en saison sèche et 0,001 et 0,047 méq/100 $\mathrm{g}$ en fin de saison des pluies. Même si les valeurs de sodium sont globalement faibles dans l'ensemble des cuvettes (littorales et continentales), il est noté une hausse de sa teneur en saison sèche (Fig. 9). Les villages du secteur continental enregistrent des valeurs de sodium de moins de 0,1 méq/100 g. 


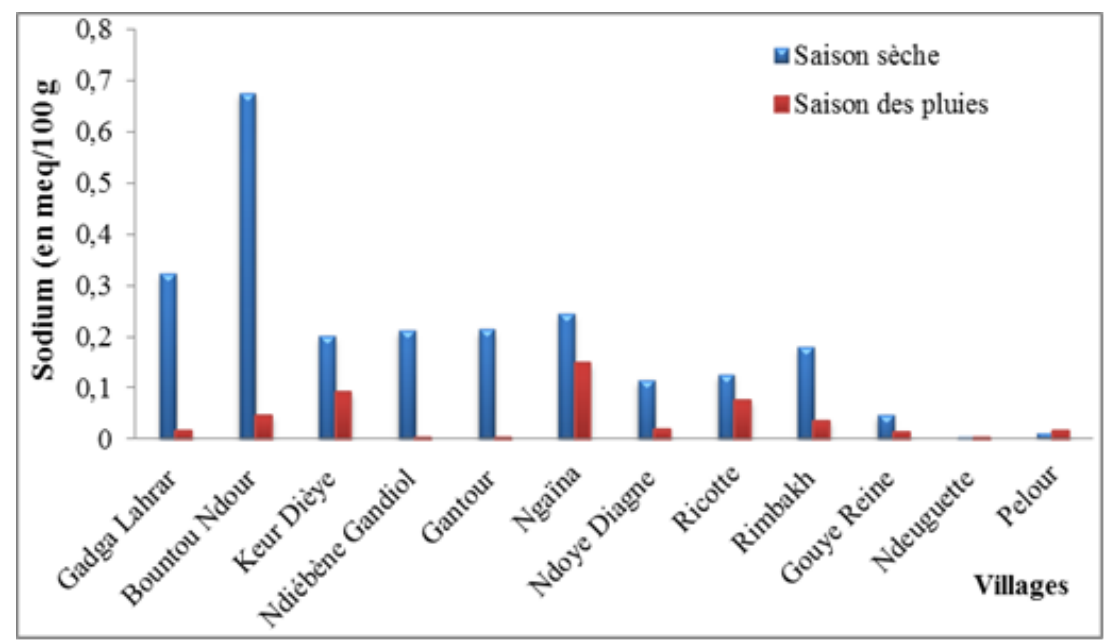

Figure 9 : Variation du sodium $(\mathrm{Na})$ en fonction des saisons

Au regard de ces observations, la sodicité de l'eau d'irrigation ne pose vraisemblablement pas de problème pour ces cuvettes exploitées. De même que les pourcentages respectifs de sodium échangeable (PSE) sont très faibles (moins de $1 \%$ ) à la fois dans le secteur continental et le secteur littoral et permettent de justifier la faible concentration de sodium dans le sol des niayes. Le PSE est de 0,20 \% à Gadga Lahrar, 0,48 \% à Ndiébène Gandiol, 0,07 \% à Pelour, 0,4\% à Rimbakh Gandiol, 0,01 \% à Gantour, 0,02 \% à Ndeuguette, $0,92 \%$ à Bountou Ndour, 0,10 à Ndoye Diagne et 0,12 à Gouye Reine.

D'un autre côté, les rapports entre les cations dominants sont également caractérisés par une concentration des ions bivalents alors que les rapports $\mathrm{Na} / \mathrm{K}$ ne dépassent pas $0,6 \mathrm{méq} / 100 \mathrm{~g}$ en saison sèche et 0,320 méq/100 $\mathrm{g}$ de sol dans tous les horizons.

Les faibles valeurs de sodium et des SAR calculés montrent que la salinité n'est pas liée au complexe avec un risque moindre de sodisation. Entre les deux saisons, le SAR n'atteint pas 1. En saison sèche, les horizons respectifs $(0-15$ et $15-30 \mathrm{~cm})$ sont caractérisés par des valeurs de l'ordre de 0,222 et 0,148 à Gadga Lahrar, 0,249 et 0,158 à Bountou Ndour, 0,201 et 0,046 à Ndiébène Gandiol, 0,57 et 0,202 à Gantour, 0,088 et 0,070 à Ndoye Diagne, 0,137 et 0,108 à Rimbakh Gandiol, 0,072 et 0,074 à Keur Dièye, 0,241 et 0,112 à Ngaïna, 0,068 et 0,096 à Ricotte.

Or, après la phase de lessivage, les SAR connaissent une baisse liée logiquement aux variations des trois cations $\mathrm{Na}^{+}, \mathrm{Ca}^{+}$et $\mathrm{Mg}^{+}$. Les valeurs sont estimées à 0,006 et 0,014 à Gadag Lahrar, 0,034 et 0,031 à Bountou Ndour, 0,019 et 0,024 à Ndiébène Gandiol, 0,001 et 0,002 à Gantour, 0,012 et 0,024 à Ndoye Diagne, 0,019 et 0,030 à Rimbakh, 0,009 et 0,007 à Gouye Reine, 0,002 et 0,002 à Ndeuguette, 0,012 et 0,009 à Pelour 1 . Il n'y a pas de remplacement du $\mathrm{Ca}^{+}$par les $\mathrm{Na}^{+}$à partir du moment où les valeurs de cette 
dernière sont faibles pendant les deux saisons. Il y a quasiment une absence de fixation du sodium dans le complexe argilo-humique essentiellement sableux.

La comparaison des deux faciès montre une grande différence entre les éléments présents sur l'eau et le sol (Fig. 10).



Figure 10 : Comparaison des faciès d'eau et de sol

Les sols sont essentiellement magnésiens et calciques en toute saison. Or, la composition de l'eau d'irrigation est plus concentrée combinant des cations et des anions dont $\mathrm{Na}^{+}$domine pour les cations et $\mathrm{Cl}^{-}$pour les anions. Les sels présents sont tous solubles dans la solution ce qui, toutefois, augmente les risques de sur-salinisation de la nappe d'eau douce du Gandiolais associés aux nitrates contenus dans les engrais utilisés pendant la production maraichère. Les nitrates sont compris entre 32,67 à 198,19 mg/l avec respectivement Gantour (37,6 mg/l), Gouye Reine $(69,13 \mathrm{mg} / \mathrm{l})$, Mouit (71,63 mg/l), Mboumbaye (74,33 mg/l), Ndoye Diagne (49,79 mg/l), Rao Peul (32,67 mg/l), Ricotte (86,28 mg/l), Rimbakh (198,19 mg/l), Toug Peul $(32,82 \mathrm{mg} / \mathrm{l})$.

\section{IV - Discussion}

À l'échelle des cuvettes maraichères observées, l'évolution de la minéralisation des eaux d'irrigation ne reflète pas nécessairement celle du sol. Le facteur textural est important dans la dynamique de conservation des sels dans les cuvettes agricoles. Le pourcentage de sables y est très élevé et varie entre 84,91 et 99,93\%. À partir de ce constat, on peut retenir que la présence des sels très solubles dans les niayes a un caractère transitoire compte tenu de la texture essentiellement sableuse, donc poreuse (très filtrant). 
Malgré l'importance du sel, les sols irrigués ne retiennent pas tout le sel incorporé dans l'eau de la nappe à cause de leur caractère de sol Dior (sols ferrugineux tropicaux non lessivés) qui en permet leur lessivage. Ainsi, les eaux chargées en sels alimentent-t-elles la nappe qui s'écoule dans le sens de la pente en drainant les sels vers les points les plus bas et caractérisant la forte concentration saline dans les secteurs déprimés localement appelés "Khour".

En revanche, les fractions fines (argiles et limons) sont moins présentes dans le sol (0,07 à 15,10\%). Les profils, qu'ils soient situés au niveau continental ou littoral, sont donc tous caractérisés par des sols sableux. Ces résultats sont corroborés par les travaux de O. NDIAYE (2012) et M. DIALLO et al., (2015) à partir de quelques profils pédologiques. L'observation de plusieurs profils entre Gantour et Gadga Lahrar démontre qu'à plus de $10 \mathrm{~m}^{5}$ de profondeur le faciès est homogène avec quelques intercalations argileuses, ce qui permet aux sels d'être dissous assez rapidement dans la solution du sol.

Ces observations épousent les mêmes profils litho-stratigraphiques effectués par C.H. KANE (1995) dans la zone méridionale des Niayes qui constitue un même système lithologique avec le Gandiolais. La couche de sable y atteint 20 à $80 \mathrm{~m}$ d'épaisseur. Les sols sableux montrent une vitesse élevée d'écoulement capillaire mais une hauteur limitée de remontée. Ce qui suppose que pour une quantité d'eau utilisée dans une parcelle maraichère, la proportion de sels qui arrive en surface ou en sub-surface est inférieure à celle lessivée pendant la saison des pluies. Ce principe d'écoulement et de remontée s'adosse à la comparaison des faciès de sol et des eaux (Fig. 10) qui permet de constater que la nature de l'eau d'irrigation ne conditionne pas forcément le sol irrigué dans le Gandiolais.

Les résultats démontrent aussi que les sels sont plus concentrés dans l'eau d'irrigation que le sol irrigué. Cela suppose qu'il y a une perte de ces sels transmis aux niayes par voie de lixiviation en saison des pluies. Le lien est étroit entre la présence des sels et la nature de l'encaissant surtout s'il s'agit essentiellement de sels plus solubles que le gypse. ${ }^{6}$ Mais, la dégradation est présente en atteste certaines parcelles de Ndiébène ou Gadga dont les CE atteignent $700 \mu \mathrm{s} / \mathrm{cm}$ et les $\mathrm{pH}$ sont très alcalins.

\footnotetext{
${ }^{5}$ Ces observations ont été permises lors des séances de creusement de puits destinées à l'irrigation des parcelles. Des observations ont été faites à Gadga Lahrar, Rimbakh Gandiol, Gouye Reine et Gantour. En fonction de la topo-séquence dunaire, différentes profondeurs ont été obtenues mais la limite maximale observée a été de 10,5 m à Gantour (dunes ogoliennes). Egalement, les sept profils pédologiques effectués par M.D. DIALLO et al (2015) avec des dimensions de 1,5 m x 1,5 m x 1,5 m indiquent le même résultat.

${ }^{6}$ Le gypse est caractérisé par une solubilité de 2 g/l
} 
Nous avons constaté, avec les données des deux saisons, qu'il n'y a pas de salinisation avancée des parcelles maraichères. En effet, il y a plutôt une sur-salinisation de l'eau d'irrigation dans la partie littorale maraîchère contrairement à ceux qu'estiment théoriquement quelques travaux effectués dans la zone (DIENG, 2010, KANE, 2010). Ces résultats confirment les mesures de C. KANE (2010), O. NDIAYE (2012) et M.D. DIALLO (2015 a et b).

Les faciès chimiques et les CE obtenues en saison sèche et en période de fin de lessivage ne peuvent être considérés comme des valeurs critiques de minéralisation du sol. D'ailleurs, le contexte sableux (poreux) ne favorise ni une salinisation ni un abandon rapide des parcelles maraîchères. En présence de sels très solubles, les difficultés de remontée et la lixiviation rapide dans le modelé sableux façonnent un cycle transitoire de sels dans le complexe. La nappe fournit les mêmes éléments chimiques au sol à l'exception des nitrates qui proviennent essentiellement des intrants chimiques.

Compte tenu de la situation de non conservation du sel dans le complexe, il fallait comprendre deux faits : la salinité du sol et celle de la solution du sol. Pour J.H. DURAND (1983), ces deux notions sont tout à fait différentes. En effet, la salure du sol s'exprime par sa CE d'un extrait salin. Elle correspond donc à la quantité de sels solubles retenus par le sol. Ce paramètre a été étudié pendant deux saisons contrastées et dont les valeurs n'atteignent pas $1 \mathrm{~ms} / \mathrm{cm}$ (1000 $\mathrm{SS} / \mathrm{cm})$, ce qui suppose une salinité faible du sol. En revanche, la salinité de la solution du sol dépend de son humidité et c'est effectivement dans ces solutions que les spéculations (ognons, carottes, choux, etc.) cultivées puissent l'eau dont elles ont besoin. ${ }^{7}$

Fondamentalement c'est ce deuxième phénomène qui explique les rendements faibles et les abandons de parcelles dans le Gandiolais. C'est la salinité de cette solution qui s'oppose à l'absorption d'eau nécessaire pour les plantes s'expliquant par le fait que les cations sont absorbés par les spéculations maraîchères sous forme de sels solubles. Pour ce qui concerne la production et précisément dans ce que nous essayons de montrer, la notion de salinité de la solution du sol est la plus importante et plus à même d'élucider les baisses de production.

\section{V - Conclusion}

En définitive, la dégradation chimique étudiée dans les cuvettes agricoles est principalement liée à la minéralisation de la réserve souterraine. La cause de cette salinisation est principalement secondaire et reste liée aux

${ }^{7}$ Le véritable problème de l'évolution des sols irrigables est de connaitre la solution où la plante puisera l'eau qui lui est nécessaire. Cette absorption se faisant par osmose, la plante aura à fournir un travail d'autant plus important que les solutions dont elle disposera seront plus concentrées 
modifications dans les échanges latéraux entre la nappe et les eaux marines justifiant, de fait, les forts abandons de parcelles dans le secteur littoral. Certes, la présence des sels fossiles est un facteur intrinsèque mais cette vulnérabilité naturelle se juxtapose à des mécanismes d'échanges qui sont plus déterminants dans la caractérisation de la minéralisation actuelle des eaux souterraines.

La méthode hydrochimique a fait ressortir la nature des traceurs chimiques, ce qui a permis d'apprécier le caractère pénétrant du biseau salé dans l'aquifère quaternaire. L'approche hydrogéochimique a démontré l'efficacité, dans les études de sol, de coupler les éléments ioniques de l'eau et du sol pour caractériser l'évolution du complexe pédologique.

Le Gandiolais est, en partie, protégé par la texture de son patrimoine pédologique essentiellement sableux. C'est donc une question de qualité de l'eau d'irrigation qui se pose au système maraicher gandiolais. Au regard des résultats de sol et des échantillons d'eau, il est nécessaire de prendre en compte la qualité de l'eau dans toute politique de gestion des sols irrigués.

De ce fait, les techniques envisagées doivent répondre à un évitement de la concentration des sels dans le profil des cuvettes agricoles. Le lessivage reste efficace pour la réhabilitation des niayes abandonnées compte tenu des paramètres physiques et chimiques observés. Mais, il aura un effet plus important dans les parcelles où l'alcalinité est devenue très importante. Le lessivage par de l'eau douce d'une CE de moins de $750 \mu \mathrm{s} / \mathrm{cm}$ peut parvenir à faire descendre le sel dans la nappe quaternaire. Cela suppose deux faits : d'abord la présence d'une eau douce pour la lixiviation, puis une concentration des sels au niveau de la nappe quaternaire après le lessivage. Ce dernier est possible à condition que la nappe des sables quaternaires déjà minéralisée ne soit plus utilisée pour des besoins d'irrigation des parcelles maraîchères.

\section{References:}

1. CHKIR Najiba, RIM Trabelsi, MOHAMMED Bahir, AMMAR Friha Hadj, 2008. «Vulnérabilité Des Ressources En Eaux Des Aquifères Côtiers En Zones Semi-Arides. Étude Comparative Entre Les Bassins d'Essaouira (Maroc) Et De La Jeffara, Comunicações Geológicas, Pp : 107-121.

2. DIALLO Maimouna (2006) - Aménagements Hydrauliques Et Mutations Du Bas-Estuaire Du Fleuve Sénégal : Impacts Sur La Nappe Des Sables Dunaires Du Gandiolais. Mémoire De DEA (Chaire UNESCO), UCAD, 79 P.

3. DIALLO Mariama Dalanda, NGAMB Thierno, TINE Alfred Kouly, GUISSÉ Mamadou, Ousmane NDIAYE, SALEH Minda MAHAMAT, DIALLO Aly, DIOP Aliou, GUISSÉ Aliou (2015). Caractérisation Agropédologique Des Sols De Mboltime Dans La 
Zone Des Niayes (Sénégal). Revue Agronomie Africaine, Vol. 27, $\mathrm{N}^{\circ}$ 1, P. 57-67.

4. DIALLO Mariama Dalanda, NDIAYE Ousmane, SALEH MAHAMAT, TINE Alfred Kouly, DIOP Alioune Et GUISSE Mamadou, 2015. Étude Comparative De La Salinité De L'eau Et Des Sols Dans La Zone Nord Des Niayes (Sénégal). African Crop Science Journal, Vol. 23, N 2, P. 101-111

5. DIATTA Ibrahima 2008. «Dynamiques Des Systèmes De Productions Horticoles Et Développement Territorial Dans Les Niayes (Littoral Nord-Ouest Du Sénégal » In Territoires Et Action Publique Territoriale : Nouvelles Ressources Pour Le Développement Régional, Colloque De L'asrdlf, CRDT, Québec, 14 P.

6. DURAND Jean (1983) - Les Sols Irrigables. Étude Pédologique. Édit. Presses Universitaires De France, Collection "Agence De Coopération Culturelle Et Technique", Paris, X + 339 P.

7. GAC Jean Yves Et Al, 1990. L'intrusion Saline Des Eaux Océaniques Dans La Basse Vallée Du Fleuve Sénégal Au Cours Du $20^{\text {ème }}$ Siècle, ORSTOM/Dakar, $52 \mathrm{P}$.

8. GOUAIDIA Layachi, 2008. Influence De La Lithologie Et Des Conditions Climatiques Sur La Variation Des Paramètres PhysicoChimiques Des Eaux Souterraines D'une Nappe En Zone Semi-Aride, Cas De La Nappe De Meskiana, Nord-Est Algérien. Thèse De Doctorat, Université d'Annaba, 129 P.

9. GOUAIDIA Layachi, GUEFAIFIA Omar, BOUDOUKHA Abderahmane, LAIDHEMILA Mohamed Et MARTIN, Claude 2012. " Evolution De La Salinité Des Eaux Souterraines Utilisées En Irrigation Et Risques De Dégradation Des Sols : Exemple De La Plaine De Meskiana (Nord-Est Algérien) », Physio-Géo, Pp : 141-160.

10. HACHICHA M., M'HIRI A., BOUKSILA F., Et BACH HAMBA I., 1997. Variabilité Et Répartition De L'argile Et De La Salinité Dans Le Périmètre De Kalaât Landelous (Tunisie): Application À L'évaluation Des Risques De Salinisation. Etude De Gestion Des Sols, 4, 1, Pp: 5563.

11. HAMMIA I., 2012. Impact De L'irrigation Sur La Salinisation Des Sols Dans Les Palmeraies De L'oued Righ, Mémoire De Fin D'étude, Université KASDI MERBAH-OUARGLA, 79 P.

12. HARTANI Tarik, DOUAOUI Abdelkader, KUPER Marcel, F. HASSANI, 2007. «Stratégies De Gestion Individuelle De La Salinité Dans Le Périmètre Irrigué Du Bas Chétif : Cas Du Périmètre De Ouarizane ", Cirad/Tunisia, 16 P.

13. HICHAM B. D, 2012. Salinité Des Eaux Et Des Sols Au Niveau De La Sebkha De Bemendil, Caractérisation Et Conséquences Sur 
L'environnement, Mémoire D'ingénieur En Hydraulique, Université Kasdi Merbah, Ouargla, 94 P.

14. JACOUTOT Aurélient, 2006. Modifications Environnementales Et Ses Conséquences : Cas Du Gandiolais Et Parc National De Diawling, Stage De Recherche, IGA, 174 P.

15. KANE Cheikh Hamidou, 1995. Contribution À L'étude Hydrochimique De La Nappe Des Sables Quaternaires Du Littoral Nord Du Sénégal Entre Kayar Et Saint-Louis, Thèse $3^{\text {ème }}$ Cycle, UCAD, $162 \mathrm{P}$.

16. KANE Coura, 2010. Vulnérabilité Du Système SocioEnvironnemental En Domaine Sahélien : L'exemple De L'estuaire Du Fleuve Sénégal, Thèse De Doctorat, Université Strasbourg, 317 P.

17. KOUZANA Lamia, BEN MAMMOU Abdallah Et GAALOUL Nourredine, 2007. « Intrusion Marine Et Salinisation Des Eaux D'une Nappe Phréatique Côtière (Korba, Cap-Bon, Tunisie) », Geo-EcoTrop, Numéro 31, Pp : 57-70.

18. LOYER Jean Yves, 1991. « Dégradation Saline Des Sols, Induite Par L'irrigation En Domaine Sahélien », ORSTOM, 9 P.

19. LTIFI W., 2008. Bilan D'eau Et Des Sels Dans Les Périmètres Irriguées KALAAT L'ANDALOUS, Laboratoire Du Centre De Gestion Des Ressources En Eau (CGRE) De L'inat, Mémoire De Mastère, $120 \mathrm{P}$.

20. MARLET Serge, JOB Jean Olivier, 2006. « Processus Et Gestion De La Salinité Des Sols », In Tiercelin, J. R. Traité D'irrigation, Seconde Édition. Tec Et Doc Lavoisier, 28 P.

21. MDIKER Nabil, El ACHHEB Abderrahim, MANDOUR Abdenbi, YOUNSI Abdelkader, El MALIKI Soufiane Et OUTEYEB B Khadija, 2009. Contribution À L'étude De La Salinisation De La Nappe Côtière De Sahel, Afrique SCIENCE, Pp : 232-245.

22. NDIAYE Ousmane (2012) - Caractérisation Des Sols De La Zone Des Niayes De Pikine Et De Saint Louis (Sénégal). Int. J. Biol. Chem. Sci., Vol. 6, P. 519-528.

23. NIANG Souleymane, SY Amadou Abou SY Et SY Boubou Aldiouma, 2016. « Evolution Spatiale Et Hydrochimique De La Salinité De L'eau D'irrigation Dans Le Gandiolais, Littoral Nord Du Sénégal », Revue De Géographie Du Laboratoire Leïdi, Pp : 241-257.

24. RASSOU Khadija Kaid, 2009. Etude Des Interactions Entre Les Eaux Souterraines Et Les Eaux De Surface Dans Le Bassin Côtier D'oualidia, Thèse De Doctorat, Université CADI AYYAD, 214 P. 